\title{
On the Mass Distribution of Fragments of an Asteroid Disrupted in the Earth's Atmosphere
}

\author{
Irina G. Brykina $(\mathbb{D})$ and Lidia A. Egorova \\ Institute of Mechanics, Lomonosov Moscow State University, Moscow 119192, Russia \\ Correspondence should be addressed to Lidia A. Egorova; egorova@imec.msu.ru
}

Received 19 March 2021; Revised 19 May 2021; Accepted 12 June 2021; Published 24 June 2021

Academic Editor: Maria Gritsevich

Copyright ( 92021 Irina G. Brykina and Lidia A. Egorova. This is an open access article distributed under the Creative Commons Attribution License, which permits unrestricted use, distribution, and reproduction in any medium, provided the original work is properly cited.

\begin{abstract}
To model the interaction with the atmosphere of fragments of a disrupted asteroid, which move independently of each other, it is necessary to know their mass distribution. In this regard, an analogy is drawn with fragmentation in high-speed impact experiments performed to simulate the disruption of asteroids at their collisions in outer space. Based on the results of impact experiments and assuming a power law for the mass distribution in a differential form, we obtained the cumulative number of fragments as a function of the fragment mass $m$ normalized to the total mass of fragments, the mass fraction of the largest fragment(s), the number of the largest fragments, and the power index. The formula for the cumulative number of fragments of a disrupted body is used to describe the results of impact experiments for different fragmentation types. The proposed fragment mass distribution is also tested by comparison with the mass distributions of recovered meteorites in the cases of Mbale, Bassikounou, Almahata Sitta, Košice, and Chelyabinsk meteorite falls.
\end{abstract}

\section{Introduction}

Asteroids entering the Earth's atmosphere are disrupted under the action of aerodynamic forces, which increase as they penetrate into the denser atmosphere. Disruption of cosmic bodies is a complicated process, depending on many factors: the composition, structure, density, size, and velocity of the body and can occur in different ways. When an asteroid breaks up into a large number of fragments, at the first stage, they move with a common shock wave. To simulate meteoroid disruption at this stage, models of a cloud of fragments moving as a single body were developed [1-5] and used [6-8]; the overview of the models is given in [8]. At the second stage, fragments are scattered far enough to have their own shock waves that interact with each other [9-11]. At the third stage, after complete separation, fragments move independently. To simulate the independent motion of fragments of a destructed cosmic body, models of progressive fragmentation, continuous $[12,13]$ and discrete, and the instantaneous breakup of a meteoroid or its fragments at one or several gross-fragmentation points were proposed and used [14-18]. In recent years, complex hybrid models have been used, combining different types of fragmentation, including breaking up into large independent fragments and clusters of small fragments. Such approaches have been used to model fragmentation and light curves of Lost City, Innisfree [19], Benešov [19-21], TC3 2008 [22], Košice $[21,23]$, Chelyabinsk $[6,21,24,25]$, Maribo CM2 [26] bolides, and others.

When modeling the independent motion, ablation, and luminosity of fragments of a destructed asteroid, it is necessary to know their mass distribution. In this regard, an analogy can be drawn between the disruption of asteroids (meteoroids) in the Earth's atmosphere and the disruption of asteroids at their collisions in outer space. To study the collisional evolution of asteroids and simulate their destruction, many experiments have been carried out on the destruction of solid bodies during a high-speed impact [27-42], and others; some reviews are given in [27-29, 32, 36, 37]. Experimental studies covering a wide range of target sizes, shapes, and materials (terrestrial: rocks, cement mortar, basalt, gypsum, glass, ice, artificial 
conglomerates, etc., and meteorite samples: ordinary and carbonaceous chondrites), impact velocities, and projectile materials investigate the size, shape, rotation, velocity, and mass (size) distribution of the fragments generated by the impact.

The mass distribution of fragments of a destructed body is usually characterized by a function of the cumulative number of fragments $N_{m}(m)$, which is defined as the number of fragments with masses greater than or equal to $m$. In many experimental studies, it was noted that the cumulative mass distribution curve for some fragmentation types is well described by a power law [27-42]. This is usually represented in a simple form where the cumulative number of fragments $N_{m}$ is proportional to the power of $m$ : $N_{m}=B m^{-\beta}$, where coefficient $B$ and power index $\beta$ are treated as constant. This correlation between the function $N_{m}(m)$ and fragment mass $m$ gives a linear plot in log-log coordinates with a negative power index slope. However, as also noted in many studies [27-31, 33-37, 40-42], the whole mass distribution curve usually cannot be well represented by a single exponent in the power law and is divided into two or three segments with a steeper slope for larger fragments. In addition to the power law, statistical distributions and multimodal models for nonhomogeneous bodies are used to fit experimental data (see, for example, [43]). In this paper, we use the power law for the fragment mass distribution, presented in differential (incremental) form, which is also given in some papers on impact experiments [27-29, 31, 32]. Integrating this equation and using the mass conservation equation, we obtain the expression for the cumulative number of fragments $N_{m}(m)$, which is not a linear plot in $\log$ - $\log$ coordinate and enables us to describe the results of impact experiments with a single curve, that is, using a single exponent.

A similar approach with the use of a power law for the fundamental grain mass distribution, but using a discrete form rather than a continuous one, was used to model the light curves of Leonid meteors $[44,45]$, and it was concluded that most Leonid light curves could be fitted using a power law distribution of grains. Such approach was used in [46] to model deceleration and light curves of Draconid meteors and in other studies of small meteoroids, as well as in a hybrid model of fragmentation of large meteoroids to describe the mass distribution of small particles (dust), for example, in [23]. The power law of size distribution was also derived based on the analysis of observations of fragmentation of the HAYABUSA spacecraft main body upon its reentry into the Earth's atmosphere [47]. It was a unique opportunity to observe the fragmentation of the "artificial meteoroid." The process has been described in detail; the change in the number of fragments and their size distribution has been obtained by analyzing the images and estimating the brightness of fragments.

The power law mass frequency distribution is also used in the problem of determining meteoroid mass distribution indices for sporadic meteors and meteor showers using radar meteor observations [48-51]. It is shown [52] that the initial mass spectra of various astronomical objects (cosmic dust, asteroids, planets, stars, star clusters, galaxies, and galactic clusters) in ensembles formed by fragmentation (a fast process) can be represented in a first approximation in a statistically significant range by a basis distribution function following a power law. A power law is used in investigating the size-frequency distribution of impact craters on terrestrial planets and asteroids and of projectiles that formed those craters [53-55]. Size distributions based on a power law are widely used to describe various phenomena in geoscience [56-58]: earthquakes (sizes in terms of energy or seismic moment), forest fires areas, the energy of tropical cyclones, amount of rainfall, impact fireballs (sizes in terms of total impact energy), and other natural phenomena. An overview of power law distributions in geoscience is given in [58].

When an asteroid is disrupted in the Earth's atmosphere, the mass distribution of its fragments can be inferred to some extent by the mass distribution of meteorites that have fallen to the ground. Therefore, it seems natural to compare the proposed in this study mass distribution of fragments of a destructed body, based on the power law, with the mass distributions of recovered meteorites in cases where a sufficiently large number have been collected. Previously, various approaches were suggested to approximate the mass (size) distribution of recovered meteorites. Size distributions in six meteorite showers (Barwell, Bruderheim, Gibeon, Johnstown, Sikhote-Alin, and Tenham) were considered in [59] using some empirical formulas. Mass distributions of fragments from sixteen meteorite showers were considered in [60] using some functions, in particular a power law with exponential cutoff and scaling parameters adjusted for each shower to better agreement of theoretical distributions with empirical. The same approach, applying the power law distribution with an exponential cutoff, was used in [61-64] to estimate the shape of original object when considering Košice, Bassikounou, Almahata Sitta, and Sutter's Mill meteorite falls. A curve of the cumulative mass distribution of collected fragments from the Mbale meteorite shower was constructed in [65], and it was shown that a simple power law distribution (straight line in log-log coordinates) fitted the part of this curve for large fragments but could not describe the whole curve. For the Chelyabinsk event, it was also shown [66] that power law distribution in the form of the straight line in log-log coordinates fits only the middle part of meteorites mass distribution. A curve of the mass distribution of the Chelyabinsk meteorites was approximated in $[67,68]$ by a polynomial of the third degree in logarithmic variables. The mass distribution of recovered fragments after Košice meteorite fall was approximated in [69] using various complex statistical models having several adjusted free parameters; the most appropriate of them were bimodal Weibull, bimodal Grady, and bimodal lognormal distributions. The bimodal lognormal distribution and the more generic exponential distribution were used to model the meteoroid fragmentation in Monte Carlo simulations [70] to predict the strewn field of fallen meteorites, in particular for Košice. The exponential, bimodal exponential, $q$-exponential, and $q$-stretched exponential distributions were used in [71] to fit the Košice, Sutter's Mill, and Whitecourt meteorite mass distributions. 
Here, we test the proposed formula for the cumulative number of fragments depending on the fragment mass $m$ normalized to the total mass of fragments, the mass fraction of the largest fragment (s), the number of the largest fragments, and the power index as applied to the Mbale, Bassikounou, 2008 TC3 (Almahata Sitta), Košice, and Chelyabinsk meteorite falls.

\section{Mass Distribution of Fragments}

The mass distribution of fragments of a disrupted body is usually described by the function of the cumulative number of fragments $N_{m}(m)$ with masses greater than or equal to $m$, where $m$ is the fragment mass. In this study, we use a differential form of the power law mass distribution $[27-29,31,32]$ based on the results of impact experiments:

$$
\begin{gathered}
-\frac{\mathrm{d} N_{m}}{\mathrm{~d} m}=n_{m}, \\
n_{m}=D m^{-\beta-1} .
\end{gathered}
$$

Here, $\mathrm{d} N_{m}=n_{m} \cdot \mathrm{d} m$ means the number of fragments in the mass range from $m$ to $m+\mathrm{d} m$, and coefficient $D$ and power index $\beta$ are considered as constants. The functions $N_{m}$ $(m)$ and $n_{m}(m)$ are considered as continuous functions, defined for $m \leq m_{l}$, where $m_{l}$ is the mass of the largest fragment, or fragments if there are several largest fragments with the same mass.

Coefficient $D$ is found using the fact that the body mass $M$ (mass of the asteroid just before breakup, mass of the target in experiments) is preserved during destruction; i.e., the mass $M$ is equal to the total mass of all fragments. This can be written as the mass conservation equation:

$$
M=\int_{0}^{m_{l}} n_{m}\left(m^{\prime}\right) m^{\prime} \mathrm{d} m^{\prime} .
$$

Substituting the function $n_{m}$ from relation (2) into this equation, we can find the unknown coefficient $D$ as a function of parameters $M$ and $m_{l}$, which we consider as fixed and known values, and power index $\beta$ (here, we assume $0<\beta<1)$ :

$$
D=M \frac{1-\beta}{m_{l}^{1-\beta}} .
$$

Then, using Equations (2) and (4), we obtain the formula for the function $n_{m}(m)$ :

$$
n_{m}=M \frac{1-\beta}{m_{l}^{1-\beta}} m^{-\beta-1}=\frac{1-\beta}{M l^{1-\beta}}\left(\frac{m}{M}\right)^{-\beta-1},
$$

where $l=m_{l} / M$ is the dimensionless mass of the largest fragment. The mass fraction of the largest fragment $l$ is an important parameter characterizing the degree of fragmentation.

To find the cumulative number of fragments $N_{m}(m)$, we integrate Equation (1) from $m$ to $m_{l}$ :

$$
N_{m}(m)-N_{m}\left(m_{l}\right)=\int_{m}^{m_{l}} n_{m}\left(m^{\prime}\right) \mathrm{d} m^{\prime}, \quad 0<m \leq m_{l} .
$$

Let us denote the number of fragments with the largest mass $m_{l}$ by $n_{l}$. Then, $N_{m}(m)$ is found from

$$
N_{m}(m)=\int_{m}^{m_{l}} n_{m}\left(m^{\prime}\right) \mathrm{d} m^{\prime}+n_{l}
$$

Taking into account equation (5), we obtain the following formula for the cumulative number of fragments $N_{m}$ :

$$
N_{m}=\frac{1-\beta}{\beta l^{1-\beta}}\left(\left(\frac{m}{M}\right)^{-\beta}-l^{-\beta}\right)+n_{l} .
$$

Assuming that there is only one largest fragment, which is consistent with the results of most experiments, we get

$$
N_{m}=\frac{1-\beta}{\beta l^{1-\beta}}\left(\left(\frac{m}{M}\right)^{-\beta}-l^{-\beta}\right)+1 .
$$

Thus, we have obtained the cumulative number of fragments $N_{m}$ as a function of the dimensionless fragment mass $m / M$, the mass fraction of the largest fragment (s) $l$, the number of the largest fragments $n_{l}$, and the power index $\beta$.

The power index $\beta$ depends to some extent on the material of the disrupted body and on the degree of its fragmentation. In [31], the $\beta$ values determined by many researchers in impact experiments were summarized; most of the values are in the range of about $0.5-1$. Relatively low $\beta$ values, typically $0.5-0.7$ for rocks subjected to a single impact, were noted in [28]. Upon extensive grinding, fragment size becomes smaller and more uniform, and $\beta$ value increases.

The function $n_{m}$ (formula (5)) can be used to model the independent motion and ablation of fragments of the disrupted meteoroid. Independent motion of fragments can be considered when meteoroid breaks up into a cloud of fragments, moving first as a single body; then, at a height $h_{0}$, fragments disperse to a sufficient distance to move independently with their own shock waves. Sudden destruction (instantaneous divergence of fragments) at a height $h_{0}$ can also be considered. Then, at a height $h_{0}$, we have a swarm of independently moving fragments with the number of fragments in the mass range from $M_{0}$ to $M_{0}+\mathrm{d} M_{0}$ equal to $n_{m 0} \cdot \mathrm{d} M_{0}$, where

$$
\begin{aligned}
n_{m 0} & =\frac{1-\beta}{M_{e} l^{1-\beta}} m_{0}^{-\beta-1}, \\
m_{0} & =\frac{M_{0}}{M_{e}} .
\end{aligned}
$$

Here, $M_{0}$ is the fragment mass at $h_{0}, m_{0}=M_{0} / M_{e}$ is the normalized fragment mass at $h_{0}, M_{e}$ is the total mass of fragments at $h_{0}$, and $l$ is the dimensionless mass of the largest fragment at $h_{0}$.

To find the total mass $M_{\Sigma}$, energy deposition per unit height $\mathrm{d} E / \mathrm{d} h_{\Sigma}$, and luminosity $I_{\Sigma}$ of a fragmented meteoroid, we need to integrate over all fragment initial masses: 


$$
\begin{aligned}
M_{\Sigma} & =\frac{1-\beta}{l^{1-\beta}} \int_{0}^{l} m_{0}^{-\beta-1} M \mathrm{~d} m_{0} \\
I_{\Sigma} & =\frac{1-\beta}{l^{1-\beta}} \int_{0}^{l} m_{0}^{-\beta-1}\left(-\tau \frac{\mathrm{d}}{\mathrm{d} t} \frac{M V^{2}}{2}\right) \mathrm{d} m_{0}, \\
\left(\frac{\mathrm{d} E}{\mathrm{~d} h}\right)_{\Sigma} & =\frac{1-\beta}{l^{1-\beta}} \int_{0}^{l} m_{0}^{-\beta-1}\left(-\frac{1}{V \sin \theta} \frac{\mathrm{d}}{\mathrm{d} t} \frac{M V^{2}}{2}\right) \mathrm{d} m_{0} .
\end{aligned}
$$

Here, $\tau$ is the luminous efficiency [16]. $M$ and $V$ are the mass and velocity of each fragment, which can be found, for example, using the meteor physics equations for a single body [72] as functions of initial parameters: $h_{0}, m_{0}, M_{e}, l$, and $V_{e}$ (the meteoroid velocity at $h_{0}$ ), the entry angle, and the meteoroid density.

\section{Comparison with Experimental Data}

Numerous impact experiments have shown that high-velocity impact phenomena can be conditionally classified into several fragmentation types. In [29, 31, 32], four fragmentation types are described, depending mainly on the specific energy, which is defined as the kinetic energy of the projectile per unit target mass, and also on the target material and shape, impact geometry, etc. At high specific energy, a large number of small particles occur: high degree or catastrophic fragmentation. At a lower specific energy, relatively large and medium-sized fragments are present together with small ones: cone type of fragmentation. The regime was found when there is one main fragment, which is much larger than the others: the core type of fragmentation. Destruction was also observed when only a crater was formed on the target surface and no spallation occurred: cratering type. This classification is also followed in many other experimental works, for example, in [30, 33-35, 39, 40].

A slightly different classification of disruption types depending on the degree of fragmentation of the target is given in [41]. Type I is cratering. Type II is a transition type where parts of the side surfaces are chipped off. Type III is the core type, which is signified by the whole surface of the target being spalled off with only the core in the central part of the target remaining. Type IV is complete destruction, in which the targets are completely destroyed into fine fragments and no core is left. Types III and IV are called in [41] catastrophic disruption (specific energy more than $1.05 \mathrm{~kJ} /$ $\mathrm{kg}$ ), while types I and II are called noncatastrophic disruption.

We compared the proposed fragment mass distribution with the results of high-speed impact experiments on targets of different materials [30, 31, 33-35, 40, 41] for complete and core types of destruction when using the classification [41] and for catastrophic, cone, and some types of core destruction when using the classification [29-32]. The core type of disruption was observed both for targets with homogeneous structure [29, 31, 32, 35, 41] and for inhomogeneous targets [33, 39]. In [39], different types of core destruction were studied, and it was noted that in the case where the largest core fragment is one order of magnitude larger than the second fragment and there are many small fragments, the distribution curve formed by fragments smaller than or equal to the second one looks like a typical curve of catastrophic disruption and obeys power law mass distribution. When calculating the cumulative number of fragments by formula (8) for a cone or core destruction, where the largest fragment(s) is several times larger than the next one, we excluded the largest fragment(s) from the calculation and started from the second (third) one. Some comparisons of the results of impact experiments with theoretical curves are presented in Figures 1-3 .

Figure 1 shows the comparison of the proposed fragment mass distribution with the results of impact experiments on spherical porous gypsum targets [40] for the catastrophic type of destruction (specific energy: $45.9 \mathrm{~kJ} / \mathrm{kg}$ ), which is characterized by a large number of small fragments with a uniform change in size without gaps. The mass fraction of the largest fragment is equal to 0.026; a cylindrical nylon projectile, much smaller than the target, was used in impact. In calculation by formula (9), plotted in Figure 1 by the blue line, the power index $\beta$ was adjusted to match the experimental data shown by the black dots; the results are presented in logarithmic coordinates. The cumulative mass distribution described by formula (9) is not a linear plot in logarithmic coordinates, and this makes it possible to describe the experimental mass distribution of fragments of a destroyed target by a single curve, using a single value of the power index $\beta$ (see Figure 1). This curve has an asymptote at small values of masses with a slope of the power index.

The validity of formula (9) is also supported by comparison with the mass distributions of fragments produced in four impact fragmentation experiments [30] for cubic basalt and pyrophyllite targets (see Figure 2). In Figure 2(a), the mass of both aluminum cylindrical projectiles was $10 \mathrm{~g}$, the basalt target mass was $28.73 \mathrm{~g}$, velocity was $303 \mathrm{~m} / \mathrm{s}$, and specific impact energy was $16 \mathrm{~kJ} / \mathrm{kg}$; the pyrophyllite target mass was $198.11 \mathrm{~g}$, velocity was $620 \mathrm{~m} / \mathrm{s}$, and specific energy was $10 \mathrm{~kJ} / \mathrm{kg}$. In Figure 2(b), for the basalt target with a mass of $591.4 \mathrm{~g}$, projectile mass was $2.28 \mathrm{~g}$, velocity was $950 \mathrm{~m} / \mathrm{s}$, and specific energy was $1.7 \mathrm{~kJ} / \mathrm{kg}$; for the pyrophyllite target with a mass of $159.29 \mathrm{~g}$, projectile mass was $10 \mathrm{~g}$, velocity was $289 \mathrm{~m} / \mathrm{s}$, and specific energy was $2.6 \mathrm{~kJ} / \mathrm{kg}$.

The mass fraction of the largest fragment decreases with increasing specific impact energy, and the value of the power index depends on the degree of fragmentation (see Figure 2), which, in turn, also depends on the specific energy; this was noted in various experimental studies. For both fragmentation regimes presented in Figures 2(a) and 2(b), calculations by formula (9) are in good agreement with the results of the experiments.

An example of testing the formula for the cumulative number of fragments for the core type of fragmentation (type III [41]) is shown in Figure 3. We compared the analytical calculations with the results of various experiments [41] for different sizes and shapes (cube, sphere, parallelepiped) of basalt targets. Some comparison results are presented in Figure 3 for a series of four experiments 


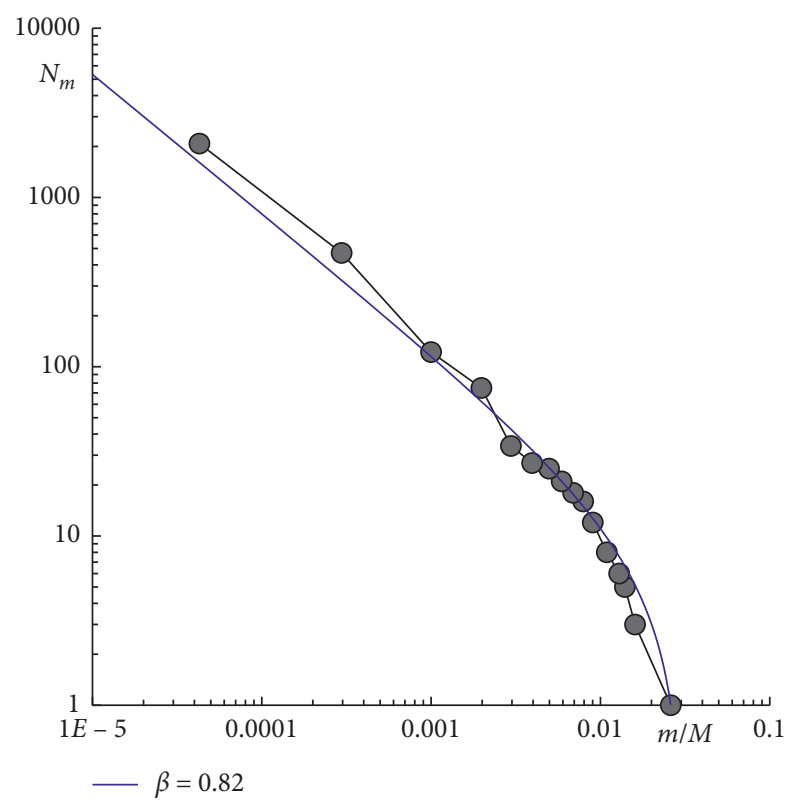

Figure 1: Cumulative number of fragments versus normalized fragment mass. Experimental results [40] at catastrophic destruction (black dots) and calculation by formula (9) at $(l)=0.026, \beta=0.82$ (blue line).

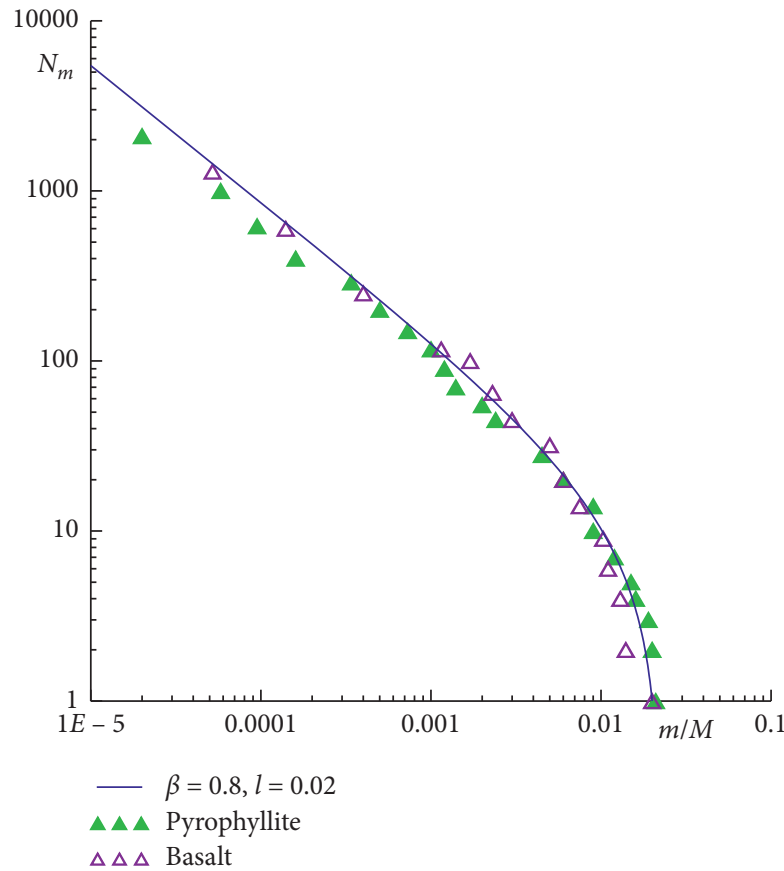

(a)

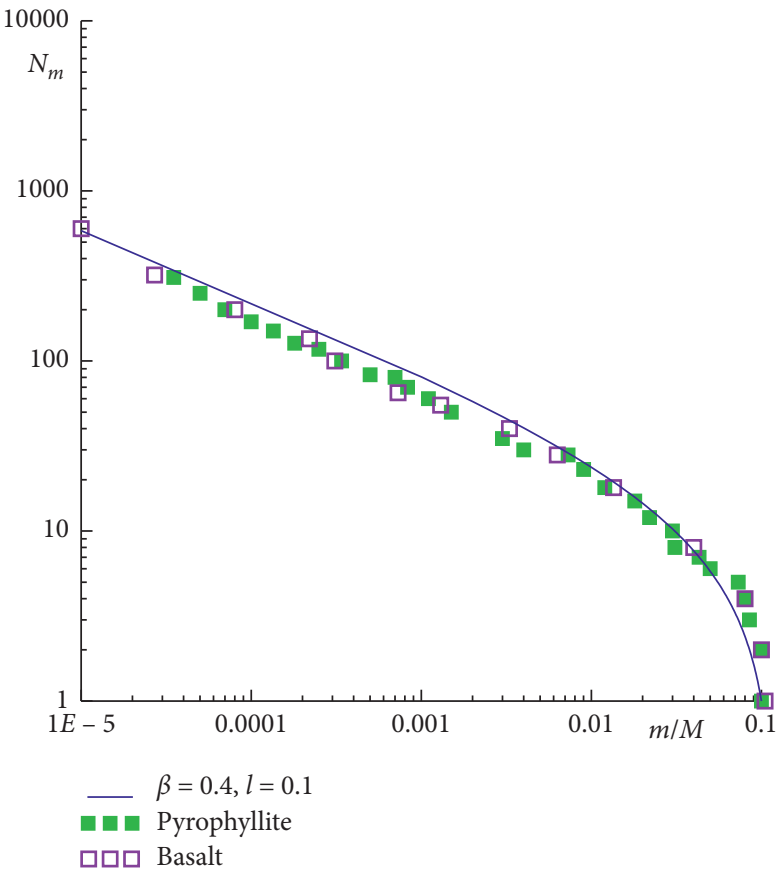

(b)

Figure 2: Cumulative number of fragments versus normalized fragment mass. Experimental results [30] are plotted by markers, calculations using formula (9) by the blue line. (a) Specific energy is 16 and $10 \mathrm{~kJ} / \mathrm{kg}$ for basalt and pyrophyllite targets. (b) Specific energy is 1.7 and $2.6 \mathrm{~kJ} / \mathrm{kg}$ for basalt and pyrophyllite targets.

carried out under nearly identical conditions to confirm the reproducibility of the experimental results. In these experiments, projectiles were shot at cubic targets with $5 \mathrm{~cm}$ side length at impact velocities of $3.7 \mathrm{~km} / \mathrm{s}$.

In three impacts, there is one largest fragment, which is much greater than the next, and in one impact, there are two largest fragments. The mass fraction of the largest fragment $l=0.092,0.065,0.049+0.038,0.061$. We used the formula (9) starting from the second fragment in Figures 3(a), 3(b), and 3(d) and from the third in Figure 3(c). Mass fractions of the following fragments differ less than mass fractions of the largest fragments: $l_{2}=0.027,0.025,0.024, l_{3}=0.02$. Cumulative mass 


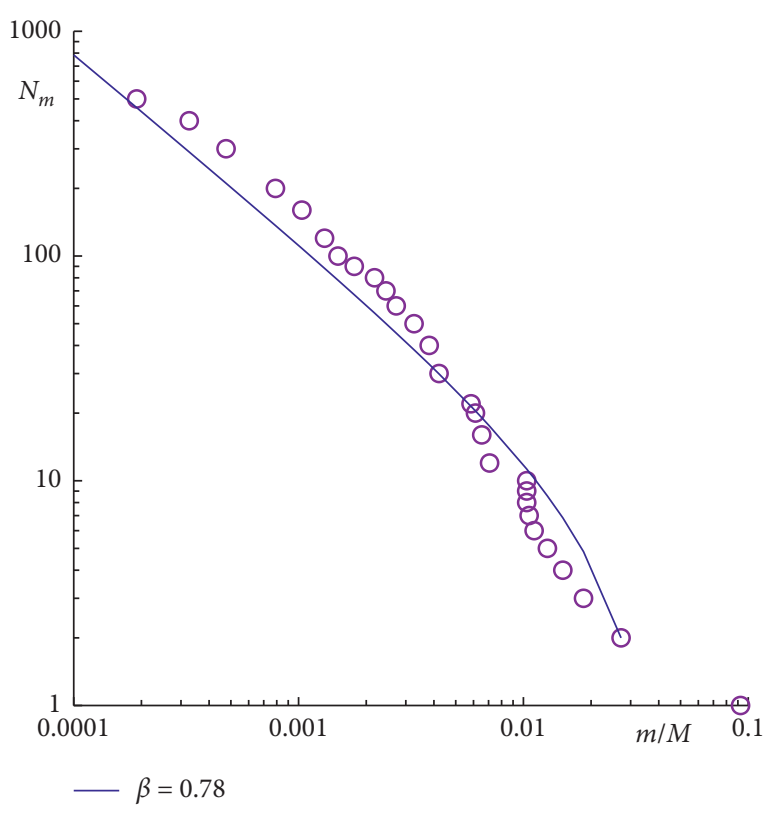

(a)

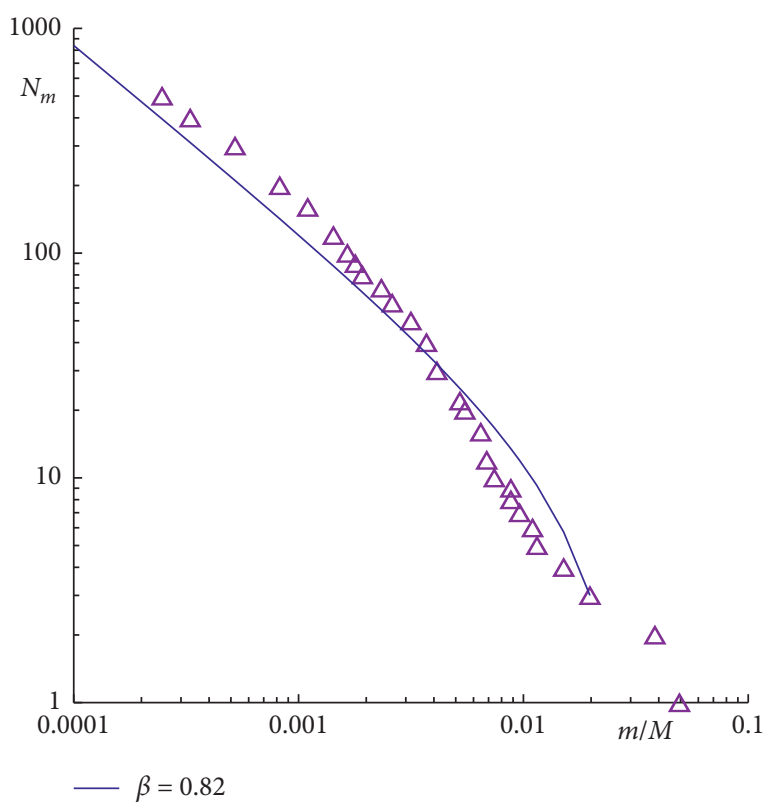

(c)

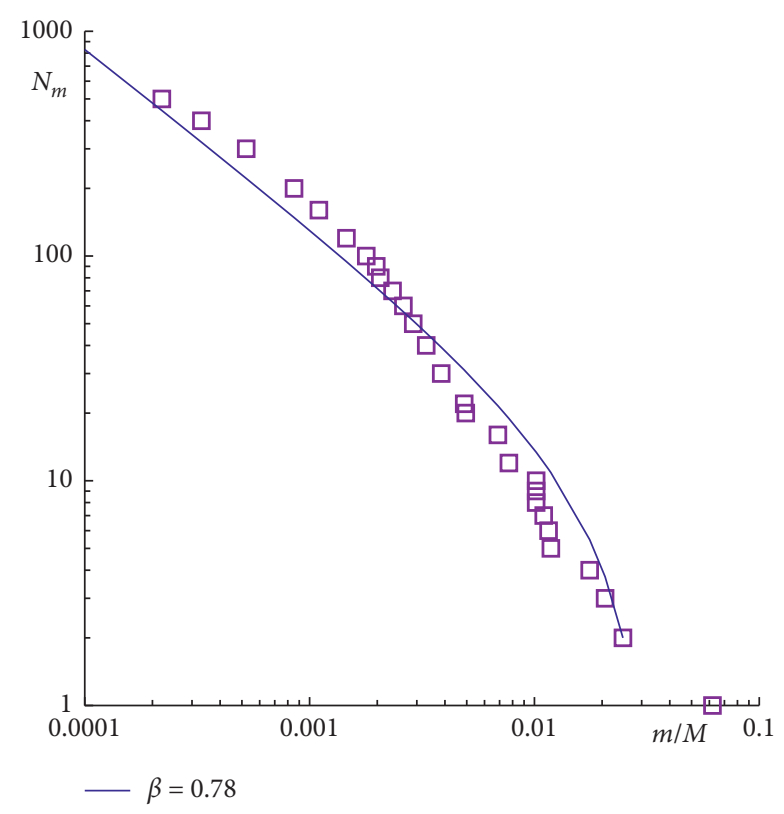

(b)

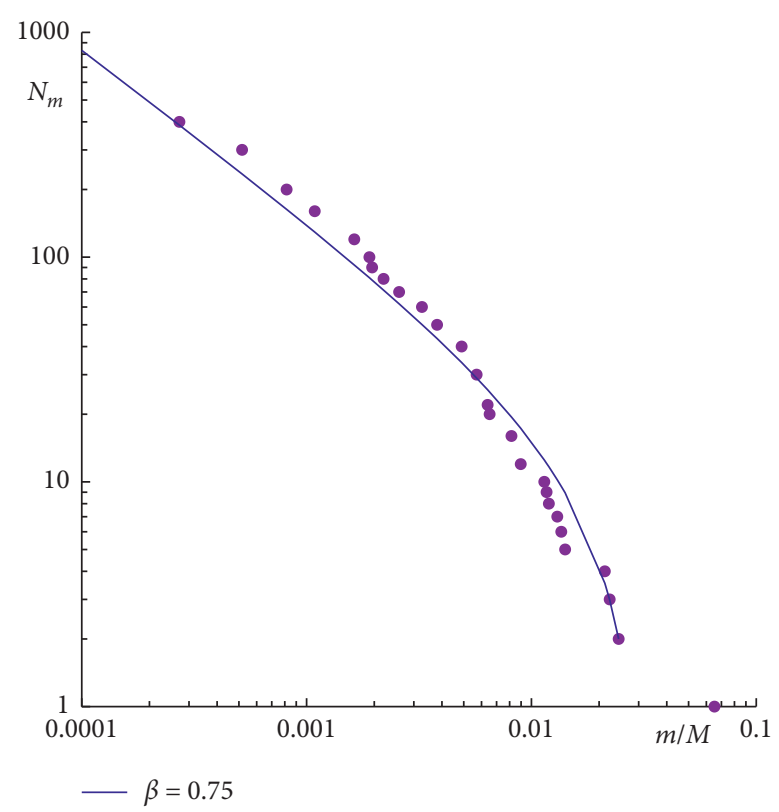

(d)

Figure 3: Cumulative number of fragments versus normalized fragment mass. Experimental results [41] at core type of destruction for four impacts under similar conditions (markers) and calculations by formula (9) (blue lines). (a) $(l)_{2}=0.027$, (b) $(l)_{2}=0.025$, (c) $(l)_{3}=0.020$, and (d) $(l)_{2}=0.024$.

distributions for four experiments shown in Figure 3 are very similar when the largest fragments are excluded (the power index changes from 0.75 to 0.82 ) and are described by the theoretical curves. In an experimental study [38] carried out for the ordinary L6 chondrite, it was also noted that when the largest one or two size fractions for each experiment are ignored, all of the distributions are remarkably similar in shape.

Comparisons with impact experiments showed that formula (8) describes the mass distribution of fragments of a destroyed body in cases of uniform change of fragment masses without gaps. In cases where there are one or two largest fragments, which are several times greater than the next one, formula (8) describes the mass distribution starting from the second (third) fragment. Note that we cannot expect that simple formula (8) with one free parameter, the power index $\beta$, can describe all the different types of destruction of bodies into many fragments, which is of a complex nature and depends on various factors. Clarification of the range of applicability of the obtained formula requires further research. 


\section{Recovered Meteorite Distributions: Results and Discussion}

We test the proposed formula for the cumulative number of fragments as a function of the normalized fragment mass by comparing it with the mass distributions of recovered meteorites in cases where a sufficiently large number of them have been collected. Here, we consider five meteorite showers.

4.1. Mbale. The Mbale meteorite fall occurred on August 14, 1992, in and around the city of Mbale in Uganda. The results of an expedition, which was held in the period from August 29 to September 5, 1992, to study the strewn field of the Mbale meteorites, which were classified as ordinary L5/6 chondrites, are presented in paper [65]. During the expedition, eye witness accounts were gathered, 48 meteorite impact positions were located, and masses between $0.1 \mathrm{~g}$ and $27.4 \mathrm{~kg}$ were recovered, which is considered to be a significant part of the total fallen mass. The certain recovered mass, including 437 fragments $(26.18 \mathrm{~kg})$, mostly small, bought from the local people by dealers, is estimated at about $150 \mathrm{~kg}$ [65].

To construct the mass distribution of the recovered meteorites, we used the available data from the paper [65] on masses of 53 individual fragments with a total mass $M=110.6 \mathrm{~kg}$, a mass of the largest fragment $m_{l}=27.4 \mathrm{~kg}$, and a mass fraction of the largest fragment $l=m_{l} / M=0.248$. The cumulative mass distribution of meteorites is shown by violet dots in logarithmic coordinates in Figure 4.

The mass distribution of meteorites is compared with calculation by formula (9) shown by the blue line; the value of the power index $\beta$ is equal to 0.4 . The calculation by the formula for the cumulative number of fragments is consistent with the distribution of Mbale meteorites with the exception of the smallest particles.

4.2. Bassikounou. The Bassikounou meteorite fall occurred on October 16, 2006, near the Bassikounou village in southeast Mauritania. Here, we use information about recovered meteorites gathered by Buhl and Baermann [73] from local Mauritanian citizens, mineral dealers, curators, and collectors. The descriptive catalog [73] describes in numbers and in images 108 single masses of a combined weight of $62.38 \mathrm{~kg}$ individually and another 382 single masses of a combined weight of $31.66 \mathrm{~kg}$ in 8 lots.

When constructing the mass distribution of the recovered meteorites, we used 108 individual masses from the catalog [73] with the total mass $M=62.38 \mathrm{~kg}$, the mass of the largest fragment $m_{l}=6.1 \mathrm{~kg}$, and the mass fraction of the largest fragment $l=m_{l} / M=0.098$. This distribution is shown by violet dots in logarithmic coordinates in Figure 5 .

The mass distribution of meteorites is compared with calculation by formula (9) shown by the blue line; the value of the power index $\beta$ is equal to 0.4 . The proposed formula for cumulative mass distribution describes the distribution of Bassikounou meteorites with masses greater than several tens of grams. If we knew the masses of 382 unaccounted

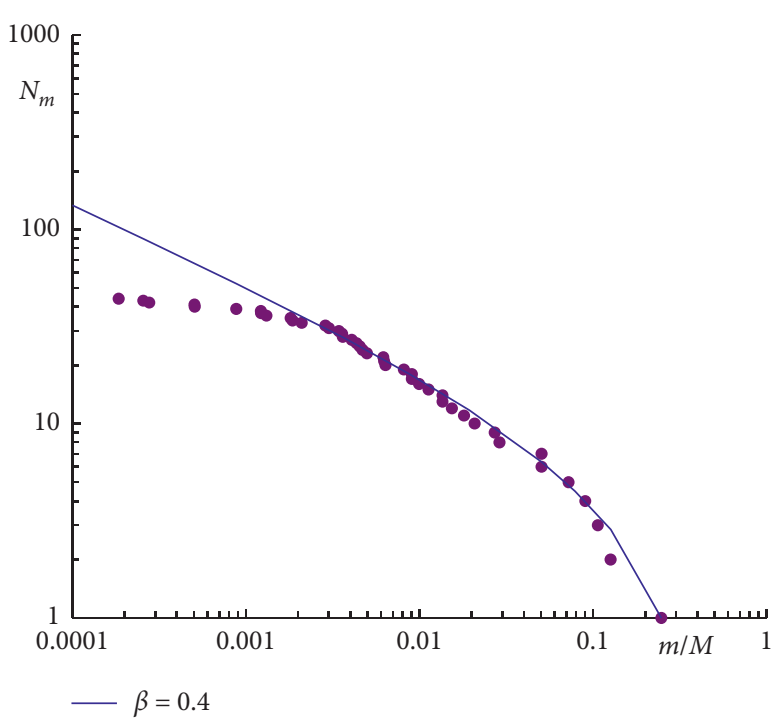

FIgURE 4: Cumulative number of fragments versus normalized fragment mass for Mbale meteorites. Violet dots: catalogue data [65], blue line: formula (9) at $\beta=0.4 ; 53$ fragments, $M(M)=$ $110.6 \mathrm{~kg}$, and $m_{l}=27.4 \mathrm{~kg}$.

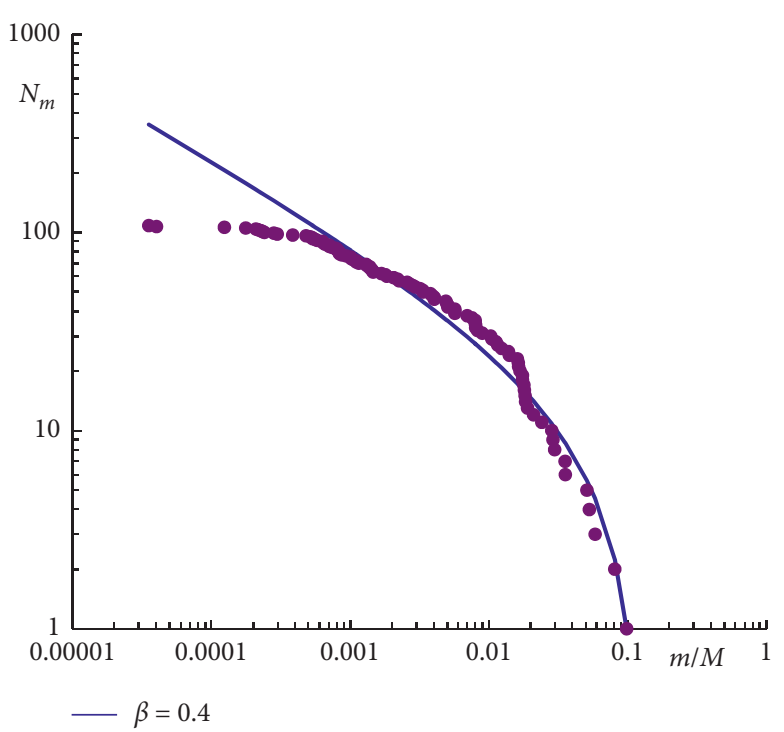

FIgURE 5: Cumulative number of fragments versus normalized fragment mass for Bassikounou meteorites. Violet dots: catalog data [73], blue line: formula (9) at $\beta=0.4 ; 108$ fragments, $M=62.38 \mathrm{~kg}$, and $m_{l}=6.1 \mathrm{~kg}$.

fragments, 365 of which are small (average weight $47.5 \mathrm{~g}$ ), from the dealer lots and added them, then the left part of the violet dot plot would rise and agreement would be better. The same applies to the distribution of Mbale meteorites.

4.3. $2008 \mathrm{TC}_{3}$ (Almahata Sitta). On October 7, 2008, a small asteroid called $2008 \mathrm{TC}_{3}$ entered the Earth's atmosphere and fragmented at an altitude of about $37 \mathrm{~km}$ above the Nubian Desert in northern Sudan [74]. The authors of paper [75] describe official expeditions to the desert, during which organized searchers and individuals discovered more than 
662 mostly small meteorites with a total mass of $10.7 \mathrm{~kg}$, named "Almahata Sitta"; their fresh look and location in the strewn field, which was predicted by calculating the asteroid $2008 \mathrm{TC}_{3}$ trajectory and accounting for high-altitude winds, suggested that they were part of this asteroid. The authors note that most of the recovered meteorites are ureilites and some are ordinary, enstatite, and carbonaceous chondrites.

When constructing the mass distribution of the Almahata Sitta meteorites, we used the masses of 662 fragments from the catalog [75] with the total mass $M=10.55 \mathrm{~kg}$, the mass of the largest fragment $m_{l}=0.379 \mathrm{~kg}$, and the mass fraction of the largest fragment $l=0.036$. The meteorites distribution log-log plot is shown by violet dots in Figure 6 . Here, calculation by formula (9) at $\beta=0.67$ is shown by the blue line.

Figure 6 shows that formula (9) gives good accuracy in describing the distribution of Almahata Sitta meteorites with masses of more than ten grams and less accuracy to a mass of about one gram. Obviously, it is problematic to find all particles with smaller masses, so the difference between the analytical prediction and the distribution of found meteorites is natural.

4.4. Košice. The Košice meteorite fall occurred in eastern Slovakia on February 28, 2010. The meteoroid, with an estimated mass of $3500 \mathrm{~kg}$, entered the atmosphere at a velocity of $15 \mathrm{~km} / \mathrm{s}$ on a trajectory with a slope of $60^{\circ}$ to the horizon; the maximum brightness was reached at an altitude of $36 \mathrm{~km} \mathrm{[23].} 78$ meteorites were recovered in the predicted fall area during several official searches, and 140 meteorites, regarding which information about the masses and coordinates is received, were found by private collectors; a number of fragments were illegally taken out of Slovakia $[23,76]$. The meteorites were classified as ordinary H5 chondrites $[23,76]$.

When constructing the mass distribution of Košice meteorites, we used the masses of 218 fragments presented in catalogs $[69,76]$. The total mass of fragments $M=11.285 \mathrm{~kg}$, the mass of the largest fragment $m_{l}=2.374 \mathrm{~kg}$, and the mass fraction of largest fragment $l=0.21$. The mass distribution corresponding to these data is shown by violet dots in Figure 7(a).

In Figure 7(a), one can see a large gap between the masses of the second largest fragment of $2.167 \mathrm{~kg}$ and the third one of $0.318 \mathrm{~kg}$. There are two ways to consider this gap. The first one is to assume that there are fragments not found or not officially reported with masses in the range of $2.167-0.318 \mathrm{~kg}$. In [69], it was shown that various statistical models give a high probability of the existence of five or slightly less number of fragments, with masses within this gap. So, when assuming the existence of missing fragments, we add 5 fragments with a total mass of $2-2.5 \mathrm{~kg}$ to the gap, in this connection, $M=13.3-13.8 \mathrm{~kg}$, and $l=0.170-0.178$ (in this interval, the mass distribution plot almost is not changed). The mass distribution of meteorites with 5 added fragments is shown in Figure 7(b). We do not plot by violet dots the added fragments in Figure $7(\mathrm{~b})$, because we do not

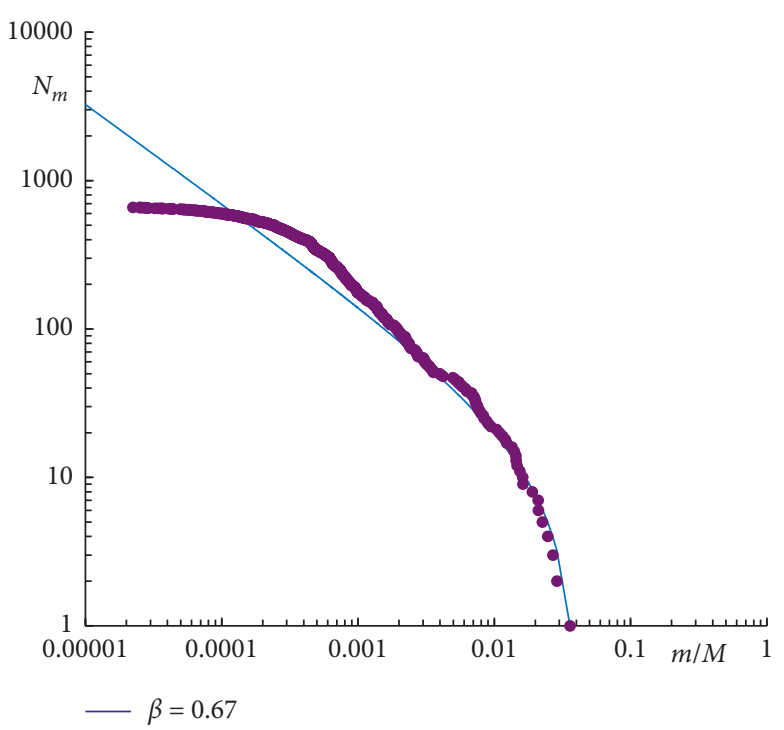

FIGURE 6: Cumulative number of fragments versus normalized fragment mass for Almahata Sitta meteorites. Violet dots: catalog data [75], blue line: formula (9) at $\beta=0.67 ; 662$ fragments, $M=10.55 \mathrm{~kg}$, and $m_{l}=0.379 \mathrm{~kg}$.

specifically define their masses. The red line corresponds to the calculation by formula (8) at $\beta=0.7$ and $n_{l}=1$.

The fall of the second fragment outside in Figure 7(b) can be explained by the existence of other missing fragments or it can be of random nature. However, the masses of the two largest fragments, $2.374 \mathrm{~kg}$ and $2.167 \mathrm{~kg}$, are close, and we can improve agreement assuming that there are two fragments with the largest mass (equal to the mean value) and applying the formula (8) at $n_{l}=2$. The mass distribution of Košice meteorites, assuming that there are two fragments with the largest mass of $2.271 \mathrm{~kg}$, is shown in Figure 7(c) (all other fragments are the same as in Figure 7(b)). Theoretical distribution is shown by the red line $(\beta=0.7)$.

Another way is to assume that there are no missing fragments and that the gap between the two largest fragments and the third one is due to the cone (or core, see section 3) type of destruction of the Košice meteoroid. The two largest fragments, which are several times larger than the next one, are characteristic of this type of destruction, often realized in experiments. Comparisons with experimental data $[30,33-35,41]$ showed that in cases where there are one or two largest fragments, which are several times greater than the next one, formula (8) describes the mass distribution starting from the second (third) fragment. Figure 7(d) shows the mass distribution of Košice meteorites starting from the third fragment, which is constructed according to the catalog data $[69,76]$, excluding the two largest fragments (in this case, $M=6.744 \mathrm{~kg}, m_{l}=0.318 \mathrm{~kg}$, and $l=0.047)$. This distribution is described by the formula for $N_{m}$ (blue line) down to a mass of fragments of a few grams.

4.5. Chelyabinsk. The passage of the Chelyabinsk meteoroid through the Earth's atmosphere on February 15, 


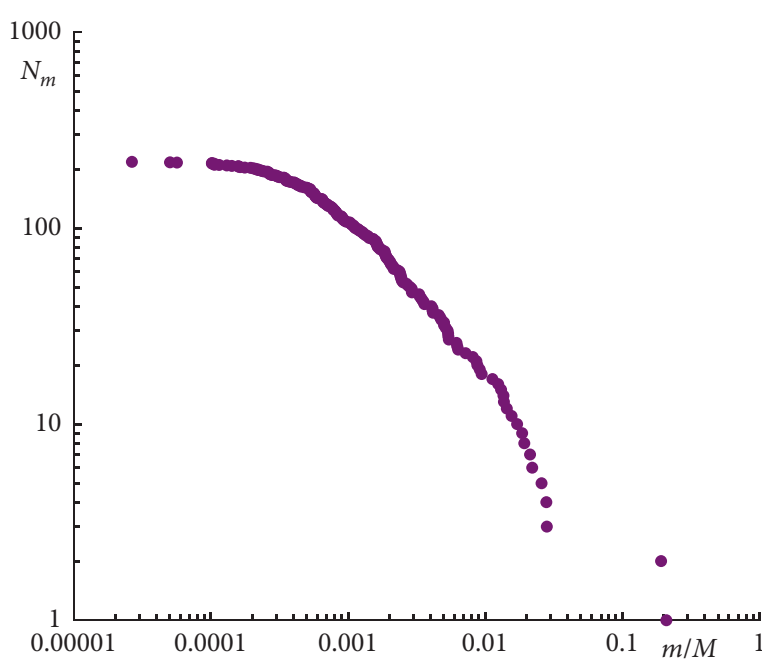

(a)

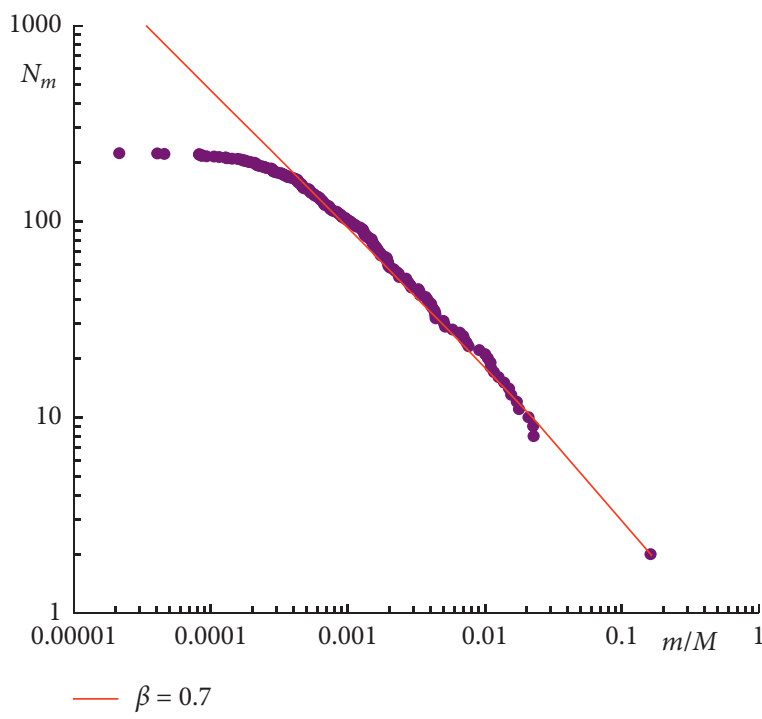

(c)

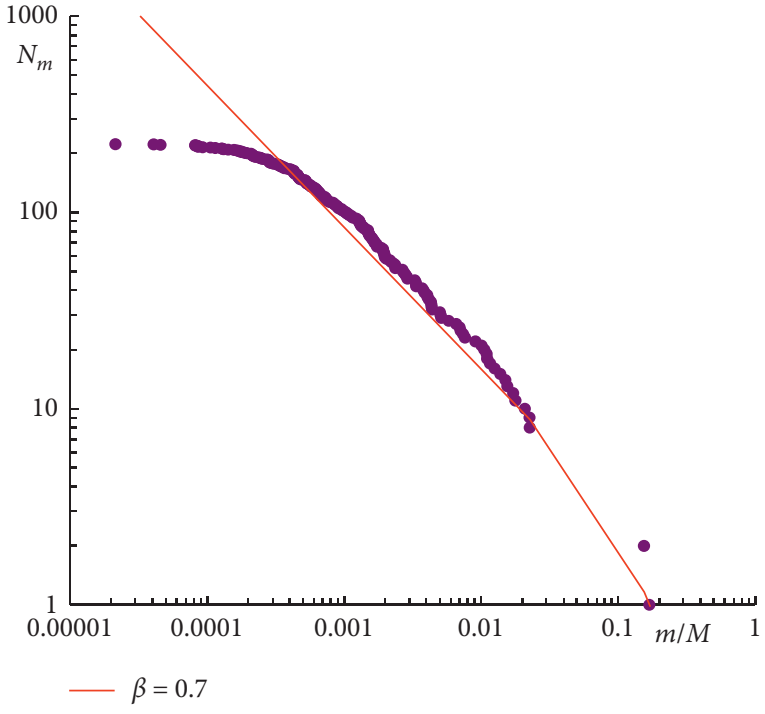

(b)

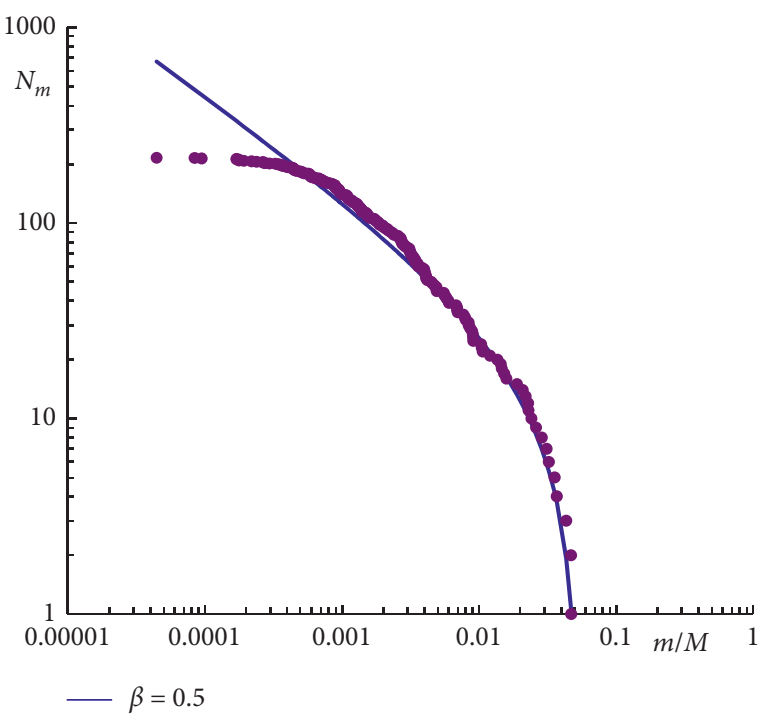

(d)

FiguRe 7: Cumulative number of fragments versus normalized fragment mass for Košice meteorites. Violet dots: catalogue data [69, 76], red and blue lines: formula (8) at $\beta=0.7$ and $0.5 ; m_{l}=2.374 \mathrm{~kg}$; (a) 218 fragments, $M=11.285 \mathrm{~kg}$; (b) 223 fragments (5 invisible), $M=13.3-13.8 \mathrm{~kg}$; (c) 223 fragments (5 invisible), two largest fragments with mass $2.271 \mathrm{~kg}, M=13.8 \mathrm{~kg}, n_{l}=2$; (d) 216 fragments, $M=6.744 \mathrm{~kg} ; m_{l}=0.318 \mathrm{~kg}$.

2013, was recorded starting from an altitude of $97 \mathrm{~km}$ by ground-based and satellite observation systems and on numerous video recordings in various localities. Based on the analysis and processing of observational data, the meteoroid trajectory, entry velocity of $19 \mathrm{~km} / \mathrm{s}$, entry angle of $18^{\circ}$, density of $3.3 \mathrm{~g} / \mathrm{cm}^{3}$, and the most probable entry mass of $1.2-1.3 \times 10^{10} \mathrm{~g}$ were determined, and meteorites were classified as ordinary LL5 chondrites $[24,25]$. Thousands of small meteorites have been recovered during many official searches, as well as by private collectors and local people. A lot of meteorites are now stored in various institutes and museums, among them the largest fragment weighing $505 \mathrm{~kg}$ from Lake Chebarkul is in the State Museum of the South Ural History.
The first data on the masses of recovered Chelyabinsk meteorites were published in [25]. First, to construct the meteorite mass distribution, we used the masses of 177 fragments presented in the study [25]. This distribution is shown by violet dots in Figure 8(a). The total mass of fragments $M=55.659 \mathrm{~kg}$, the mass of the largest fragment $m_{l}=3.4 \mathrm{~kg}$, and the mass fraction of the largest fragment $l=0.061$. Then, we added the meteorite masses from the database of Chelyabinsk State University (CSU) [77], which contain a lot of small fragments; in this connection, $M=73.889 \mathrm{~kg}, l=0.046$, and $m_{l}$ is the same, $3.4 \mathrm{~kg}$. The distribution corresponding to these data (1706 fragments) is shown by violet dots in Figure 8(b). Red, green, and blue lines in Figure (8) correspond to calculations by formula (9) 


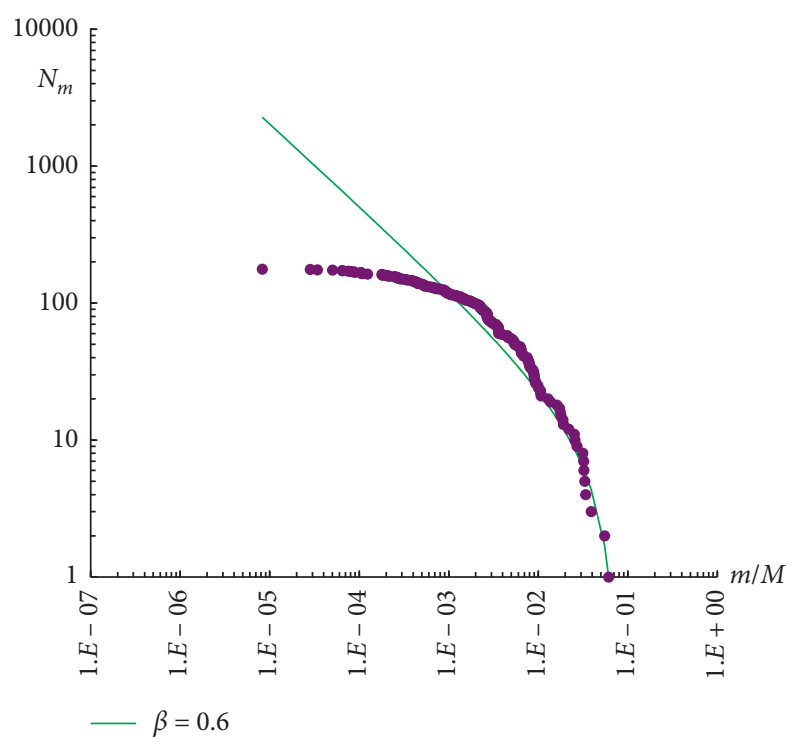

(a)

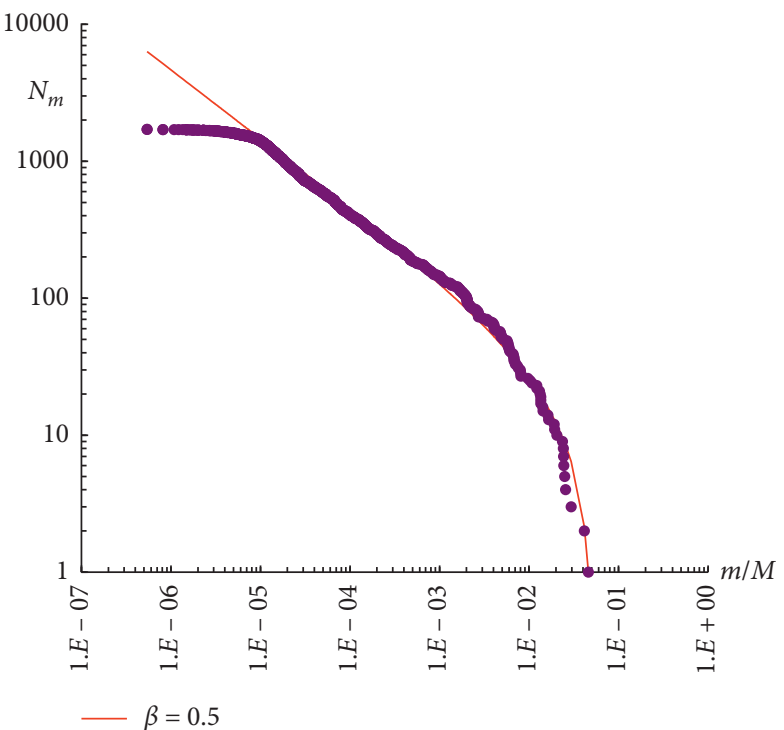

(b)

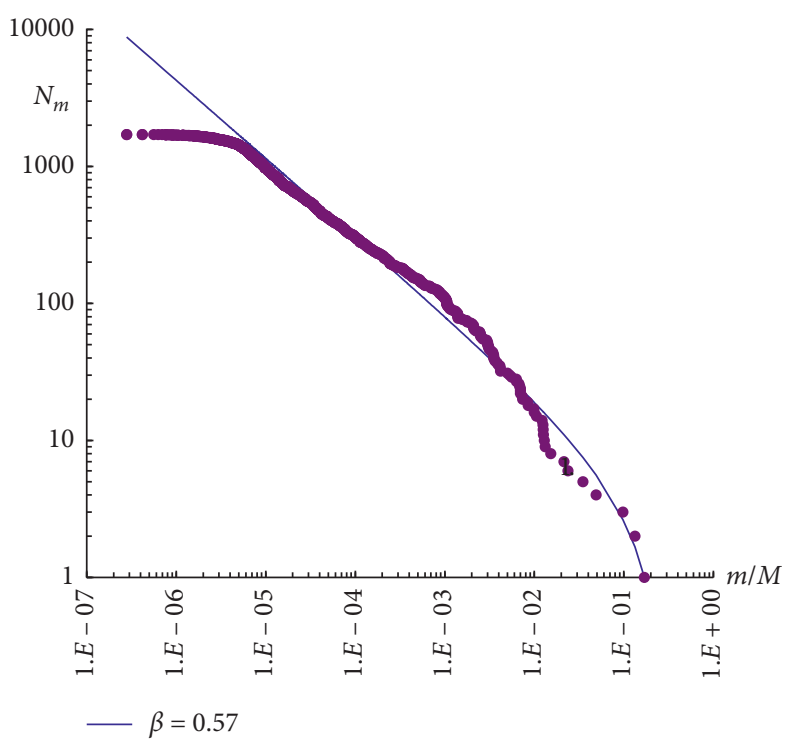

(c)

Figure 8: Cumulative number of fragments versus normalized fragment mass for Chelyabinsk meteorites. Red, green, and blue lines: formula (9) at $\beta=0.5,0.6$, and 0.57; (a) violet dots: data from [25], 177 fragments, $M=55.64 \mathrm{~kg}, m_{l}=3.4 \mathrm{~kg}$; (b) violet dots: data from [25] and Database CSU [77], 1706 fragments, $M=73.89 \mathrm{~kg}, m_{l}=3.4 \mathrm{~kg}$; (c) violet dots: data from [25], Database CSU [77], website [78], and Figure 6 [24], 1711 fragments, $M=143.19 \mathrm{~kg}, m_{l}=24.3 \mathrm{~kg}$.

at $\beta=0.5,0.6$, and 0.57 . Figures $8(\mathrm{a})$ and $8(\mathrm{~b})$ demonstrate that the addition of many small fragments from the Database CSU [77] raises the left side of the violet dots plot, as expected, and significantly improves the agreement of the calculation by formula (9) with the distribution of Chelyabinsk meteorites.

On the news website of the Chelyabinsk region "The first regional", there is information entitled "Local historians have published photos of the second largest fragment of the Chelyabinsk meteorite" that on December 1, 2013, a fragment of the Chelyabinsk meteorite weighing $24.3 \mathrm{~kg}$ was found [78], resembling in appearance and features the largest fragment found in lake Chebarkul. Several photos of this fragment are also posted on the site. Suppose that the found stone is really a fragment F2 [24] of the Chelyabinsk meteoroid. Then, it is reasonable to assume that in a large gap between $24.3 \mathrm{~kg}$ and $3.4 \mathrm{~kg}$ there may be other undiscovered or unregistered fragments, and we add in this gap the hypothetical terminal masses of observed fragments from the Extended Data in Figure 6 of the study [24]. Thus, we add to the fragments shown in Figure 8(b) a fragment weighing $24.3 \mathrm{~kg}$ [78] and four fragments between the masses of 3.4 and $24.3 \mathrm{~kg}$ from Figure 6 in [24]; in this connection, the total mass $M=143.188 \mathrm{~kg}$, and $l=0.169$. The resulting mass distribution of meteorites is shown by violet dots in Figure 8(c). 
Figure 8 shows that the addition of a large fragment with a mass of $24.3 \mathrm{~kg}$ to the collection of small fragments (mass fraction of the largest fragment increases by about 7 times) does not fundamentally change the nature of meteorite distribution. Formula (9) is in agreement with the meteorite distribution down to a mass of about a few grams.

Note that we did not include the fragment from Chebarkul Lake in the above consideration. An analogy can be drawn between the destruction of the Chelyabinsk meteoroid and the core type of destruction observed in many impact experiments both for targets with inhomogeneous structure $[33,39]$ and for homogeneous targets $[29,31,32,35,41]$. It was noted [39] that in the case where the largest core fragment is one order of magnitude larger than the second and there are many small fragments, the distribution curve, formed by fragments smaller than or equal to the second one, looks like a typical curve of catastrophic disruption and obeys a power law. Our comparisons with experimental data [30, 33-35, 41] provide support for this. The main Chelyabinsk fragment weighing $505 \mathrm{~kg}$ is about 20 times larger than the second-largest fragment weighing $24.3 \mathrm{~kg}$. Thus, we consider the mass distribution of fragments starting from the second one, which is described by formula (9). It should be noted that there are still many small fragments of the Chelyabinsk meteoroid stored in different places, which are also not included in this study.

The main problem in studying the mass distribution of recovered meteorites is that usually only a part of the fallen meteorites is recovered, and only a part of the found meteorites is officially reported. Some meteorites break up when they fall to the ground, and in some cases, it is difficult to distinguish individual meteorites and their fragments. Thus, the distribution of collected meteorites gives only an approximate picture of the real distribution of the fragments of a destructed meteoroid. Nevertheless, the above comparisons for the considered meteorite showers showed that formula (8) is able to describe the distribution of recovered meteorites for fragments with a mass more than several grams (several tens of grams) or for fragments with normalized mass $m / M>0.001$. In other words, disagreement occurs for fragments with a mass less than $0.1 \%$ of the total mass of fragments. The difference between the analytical distribution and the distribution of found meteorites at very low masses is natural because it is impossible to find all small particles. When using formula (8), the values of the adjusted power index $\beta$ for describing the distributions of meteorites were in the range from 0.4 to 0.7 and for describing the results of experiments in the range of 0.4-0.82. Further research is needed to determine more accurately the most probable value of the parameter $\beta$ or rather the range of possible values that could be used in the fragment mass distribution when considering the asteroid destruction.

\section{Conclusions}

The formula for the cumulative number of fragments of a disrupted asteroid is obtained as a function of the fragment mass normalized to the total mass of fragments (the mass of the body before destruction), the mass fraction of the largest fragment(s), the number of the largest fragments, and the free parameter: the power index. The mass distribution of fragments calculated by the proposed formula (8) is consistent with the results of high-velocity impact experiments in cases of uniform change of fragment masses without gaps. In cases where there are one or two largest fragments, which are several times greater than the next one, formula (8) describes the mass distribution starting from the second (third) fragment. The formula for the cumulative number of fragments depending on the normalized fragment mass describes the distributions of recovered meteorites constructed for Mbale, Bassikounou, $2008 \mathrm{TC}_{3}$ (Almahata Sitta), Košice, and Chelyabinsk meteorite falls with the exception of small fragments with a mass less than $0.1 \%$ of the total mass of fragments. A preliminary estimate of the most probable range of the power index of $0.4-0.7$ for meteorite distributions is given. Comparisons with empirical data give grounds to use the proposed fragment mass distribution in modeling the asteroid disruption.

\section{Data Availability}

The data used in this article are publicly available, and the article contains references to them.

\section{Conflicts of Interest}

The authors declare that they have no conflicts of interest.

\section{Acknowledgments}

This study was performed according to the research plan of the Institute of Mechanics of Lomonosov Moscow State University (Project no. AAAA-A16-116021110205-0).

\section{References}

[1] S. S. Grigoryan, "Meteorites motion and destruction in planet atmospheres," Cosmic Research, vol. 17, pp. 724-740, 1979.

[2] H. J. Melosh, "Atmospheric breakup of terrestrial impactors," Proceedings of the Seventeenth Lunar and Planetary Science, vol. 12A, pp. 29-35, 1981.

[3] C. F. Chyba, P. J. Thomas, and K. J. Zahnle, "The 1908 Tunguska explosion: atmospheric disruption of a stony asteroid," Nature, vol. 361, no. 6407, pp. 40-44, 1993.

[4] J. G. Hills and M. P. Goda, "The fragmentation of small asteroids in the atmosphere," The Astronomical Journal, vol. 105, no. 3, pp. 1114-1144, 1993.

[5] I. G. Brykina, "Large meteoroid fragmentation: modeling the interaction of the Chelyabinsk meteoroid with the atmosphere," Solar System Research, vol. 52, no. 5, pp. 426-434, 2018.

[6] P. J. Register, D. L. Mathias, and L. F. Wheeler, "Asteroid fragmentation approaches for modeling atmospheric energy deposition," Icarus, vol. 284, pp. 157-166, 2017.

[7] S. McMullan and G. S. Collins, "Uncertainty quantification in continuous fragmentation airburst models," Icarus, vol. 327, pp. 19-35, 2019.

[8] I. G. Brykina and M. .D. Bragin, "On models of meteoroid disruption into the cloud of fragments," Planetary \& Space Sci.vol. 187, Article ID 104942, 2020. 
[9] N. A. Artemieva and V. V. Shuvalov, "Motion of a fragmented meteoroid through the planetary atmosphere," Journal of Geophysical Research: Planets, vol. 106, no. E2, pp. 3297-3309, 2001.

[10] N. G. Barri, "Dynamics of two spherical objects in supersonic flow," Doklady Physics, vol. 55, no. 10, pp. 516-518, 2010.

[11] V. T. Lukashenko and F. A. Maksimov, "Numerical study on the rotation of meteoroid fragments during their separation in the atmosphere," AIP Conference Proceedings, vol. 2181, Article ID 020007, 2019.

[12] B. Baldwin and Y. Sheaffer, "Ablation and breakup of large meteoroids during atmospheric entry," Journal of Geophysical Research, vol. 76, no. 19, pp. 4653-4668, 1971.

[13] M. I. Avramenko, I. V. Glazyrin, G. V. Ionov, and A. V. Karpeev, "Simulation of the airwave caused by the Chelyabinsk superbolide," Journal of Geophysical Research: Atmospheres, vol. 119, no. 12, pp. 7035-7050, 2014.

[14] Z. Ceplecha, P. Spurný, J. Borovička, and J. Keclikova, “Atmospheric fragmentation of meteoroids," Astronomy \& Astrophysics, vol. 279, pp. 615-626, 1993.

[15] I. V. Nemchinov and O. P. Popova, "An analysis of the 1947 Sikhote-Alin event and a comparison with the phenomenon of February 1, 1994," Solar System Research, vol. 31, pp. $408-420,1997$.

[16] Z. Ceplecha, J. Borovička, W. G. Elford et al., "Meteor phenomena and bodies," Space Science Reviews, vol. 84, no. 3/4, pp. 327-471, 1998.

[17] D. O. ReVelle, "Recent advances in bolide entry modeling: a bolide potpourri," Earth, Moon, Planets, vol. 95, pp. 441-476, 2004.

[18] D. O. ReVelle, "NEO fireball diversity: energetics-based entry modeling and analysis techniques," Proceedings of the International Astronomical Union Symposium, vol. 236, pp. 95106, 2007.

[19] D. O. ReVelle and Z. Ceplecha, "Fragmentation model of meteoroid motion, mass loss, and radiation in the atmosphere," Meteoritics \& Planetary Science, vol. 40, pp. 35-54, 2005.

[20] J. Borovička, O. P. Popova, I. V. Nemtchinov, P. Spurný, and Z. Ceplecha, "Bolides produced by impacts of large meteoroids into the Earth's atmosphere: comparison of theory with observations. I. Benešov bolide dynamics and fragmentation," Astronomy \& Astrophysics, vol. 334, pp. 713-728, 1998.

[21] L. F. Wheeler, D. L. Mathias, E. Stokan, and P. G. Brown, "Atmospheric energy deposition modeling and inference for varied meteoroid structures," Icarus, vol. 315, pp. 79-91, 2018.

[22] O. Popova, "Passage of bolides through the atmosphere meteoroids: the smallest solar system bodies," in Proceedings of the Meteoroids 2010 Conference, pp. 232-242, Breckenridge, Colorado, USA, May 2011.

[23] J. Borovička, J. Toth, A. Igaz et al., "The Košice meteorite fall: atmospheric trajectory, fragmentation, and orbit," Meteoritics \& Planetary Science, vol. 48, pp. 1757-1779, 2013.

[24] J. Borovička, P. Spurný, P. Brown et al., "The trajectory, structure and origin of the Chelyabinsk asteroidal impactor," Nature, vol. 503, no. 7475, pp. 235-237, 2013.

[25] O. P. Popova, P. Jenniskens, V. Kartashova et al., "Chelyabinsk airburst, damage assessment, meteorite recovery, and characterization," Science, vol. 342, no. 6162, pp. 1069-1073, 2013.

[26] J. Borovička, O. Popova, and P. Spurný, "The Maribo CM2 meteorite fall-survival of weak material at high entry speed," Meteoritics \& Planetary Science, vol. 54, pp. 1024-1041, 2019.
[27] W. K. Hartmann and A. C. Hartmann, "Asteroid collisions and evolution of asteroidal mass distribution and meteoritic flux," Icarus, vol. 8, no. 1-3, pp. 361-381, 1968.

[28] W. K. Hartmann, "Terrestrial, lunar, and interplanetary rock fragmentation,” Icarus, vol. 10, no. 2, pp. 201-213, 1969.

[29] A. Fujiwara, G. Kamimoto, and A. Tsukamoto, "Destruction of basaltic bodies by high-velocity impact," Icarus, vol. 31, no. 2, pp. 277-288, 1977.

[30] Y. Takagi, H. Mizutani, and S.-I. Kawakami, "Impact fragmentation experiments of basalts and pyrophyllites," Icarus, vol. 59, no. 3, pp. 462-477, 1984.

[31] A. Fujiwara, "Results obtained by laboratory simulations of catastrophic impact," Memorie Della Societa Astronomica Italiana, vol. 57, pp. 47-64, 1986.

[32] A. Fujiwara, P. Cerroni, D. R. Davis et al., "Experiments and scaling laws for catastrophic collisions," in Asteroids II, pp. 240-265, University of Arizona Press, Tucson, AZ, USA, 1989.

[33] D. R. Davis and E. V. Ryan, "On collisional disruption: experimental results and scaling laws," Icarus, vol. 83, no. 1, pp. 156-182, 1990.

[34] E. V. Ryan, W. K. Hartmann, and D. R. Davis, "Impact experiments 3: catastrophic fragmentation of aggregate targets and relation to asteroids," Icarus, vol. 94, no. 2, pp. 283-298, 1991.

[35] A. Nakamura and A. Fujiwara, "Velocity distribution of fragments formed in a simulated collisional disruption," Icarus, vol. 92, no. 1, pp. 132-146, 1991.

[36] G. Martelli, E. V. Ryan, A. M. Nakamura, and I. Giblin, "Catastrophic disruption experiments: recent results," Planetary and Space Science, vol. 42, no. 12, pp. 1013-1026, 1994.

[37] K. Holsapple, I. Giblin, K. Housen, A. Nakamura, and E. Ryan, "Asteroid impacts: Laboratory experiments and scaling laws," in Asteroids III, pp. 443-462, University of Arizona Press, Tucson, AZ, USA, 2002.

[38] M. J. Cintala and F. Hörz, "Experimental impacts into chondritic targets, part I: disruption of an L6 chondrite by multiple impacts," Meteoritics \& Planetary Science, vol. 43, no. 4, pp. 771-803, 2008.

[39] C. Okamoto and M. Arakawa, "Experimental study on the impact fragmentation of core-mantle bodies: implications for collisional disruption of rocky planetesimals with sintered core covered with porous mantle," Icarus, vol. 197, no. 2, pp. 627-637, 2008.

[40] C. Okamoto and M. Arakawa, "Experimental study on the collisional disruption of porous gypsum spheres," Meteoritics \& Planetary Science, vol. 44, no. 12, pp. 1947-1954, 2009.

[41] T. Michikami, A. Hagermann, T. Kadokawa et al., "Fragment shapes in impact experiments ranging from cratering to catastrophic disruption,” Icarus, vol. 264, pp. 316-330, 2016.

[42] G. J. Flynn, D. D. Durda, E. B. Patmore et al., "Hypervelocity cratering and disruption of the Northwest Africa 869 ordinary chondrite meteorite: implications for crater production, catastrophic disruption, momentum transfer and dust production on asteroids," Planetary and Space Science, vol. 164, pp. 91-105, 2018.

[43] V. V. Silvestrov, "Application of the Gilvarry distribution to the statistical description of fragmentation of solids under dynamic loading," Combustion, Explosion \& Shock Waves, vol. 40, pp. 225-237, 2004.

[44] M. Beech and I. S. Murray, "Leonid meteor light-curve synthesis," Monthly Notices of the Royal Astronomical Society, vol. 345, no. 2, pp. 696-704, 2003. 
[45] M. D. Campbell-Brown and D. Koschny, "Model of the ablation of faint meteors," Astronomy \& Astrophysics, vol. 418, no. 2, pp. 751-758, 2004.

[46] J. Borovička, P. Spurný, and P. Koten, "Atmospheric deceleration and light curves of Draconid meteors and implications for the structure of cometary dust," Astronomy \& Astrophysics, vol. 473, pp. 661-672, 2007.

[47] J.-i. Watanabe, T. Ohkawa, M. Sato, K. Ohnishi, and Y. Iijima, "Fragmentation of the HAYABUSA spacecraft on re-entry," Publications of the Astronomical Society of Japan, vol. 63, no. 5, pp. 955-960, 2011.

[48] R. C. Blaauw, M. D. Campbell-Brown, and R. J. Weryk, “A meteoroid stream survey using the Canadian Meteor Orbit Radar - III. Mass distribution indices of six major meteor showers," Monthly Notices of the Royal Astronomical Society, vol. 414, no. 4, pp. 3322-3329, 2011.

[49] P. Pokorný, D. Vokrouhlický, D. Nesvorný, M. D. CampbellBrown, and P. G. Brown, "Dynamical model for the toroidal sporadic meteors," The Astrophysical Journal, vol. 789, 2014.

[50] P. Pokorný and P. G. Brown, "A reproducible method to determine the meteoroid mass index," Astron. \& Astroph.vol. 592, 2016.

[51] D. Janches, C. Brunini, and J. L. Hormaechea, "A decade of sporadic meteoroid mass distribution indices in the southern hemisphere derived from SAAMER's meteor observations," The Astrophysical Journal, vol. 157, 2019.

[52] A. V. Tutukov and B. M. Shustov, "Fundamental reasons for the similarity and differences of the mass spectra of various astronomical objects," Astrophysics, vol. 63, no. 4, pp. $552-565,2020$.

[53] B. A. Ivanov, G. Neukum, and R. Wagner, "Size-frequency distributions of planetary impact craters and asteroids," in Collisional Processes in the Solar System (Astrophysics and Space Science Library), vol. 261, pp. 1-34, Springer, Berlin, Germany, 2001.

[54] B. A. Ivanov, G. Neukum, W. F. Bottke, and W. K. Hartmann, "The comparison of size-frequency distributions of impact craters and asteroids and the planetary cratering rate," in Asteroids III, pp. 89-101, University of Arizona Press, Tucson, AZ, USA, 2002.

[55] M. Bronikowska, N. A. Artemieva, and K. Wünnemann, "Reconstruction of the Morasko meteoroid impact - Insight from numerical modeling," Meteoritics \& Planetary Science, vol. 52, pp. 1704-1721, 2013.

[56] S. M. Burroughs and S. F. Tebbens, "Upper-truncated power laws in natural systems," Pure and Applied Geophysics, vol. 158, no. 4, pp. 741-757, 2001.

[57] A. Deluca and A. Corral, "Fitting and goodness-of-fit test of non-truncated and truncated power-law distributions," Acta Geophysica, vol. 61, no. 6, pp. 1351-1394, 2013.

[58] Á. Corral and Á. González, "Power law size distributions in geoscience revisited," Earth and Space Science, vol. 6, pp. 673-697, 2019.

[59] M. J. Frost, "Size and spacial distribution in meteoritic showers," Meteoritics, vol. 4, no. 3, pp. 217-232, 1969.

[60] L. Oddershede, A. Meibom, and J. Bohr, "Scaling analysis of meteorite shower mass distributions," Europhysics Letters (EPL), vol. 43, no. 5, pp. 598-604, 1998.

[61] V. Vinnikov, M. Gritsevich, and L. Turchak, "Shape estimation for Košice, Almahata Sitta and Bassikounou meteoroids," Proceedings of the International Astronomical Union, vol. 10, no. 306, pp. 394-396, 2015.

[62] V. V. Vinnikov, M. I. Gritsevich, D. V. Kuznetsova, and L. I. Turchak, "Estimation of the initial shape of meteoroids based on statistical distributions of fragment masses," Doklady Physics, vol. 61, no. 6, pp. 305-308, 2016.

[63] L. I. Turchak, M. I. Gritsevich, and V. V. Vinnikov, "Statistical approach to estimate initial meteoroid shape from empirical mass distribution of recovered fragments," AIP Conference Proceedings, vol. 1773, Article ID 110014, 2016.

[64] M. Gritsevich, V. Dmitriev, V. Vinnikov et al., "Constraining the pre-atmospheric parameters of large meteoroids: Košice, a case study," Astrophysics and Space Science Proceedings, vol. 46, pp. 153-183, 2017.

[65] P. Jenniskens, H. Betlem, J. Betlem et al., "The Mbale meteorite shower," Meteoritics, vol. 29, no. 2, pp. 246-254, 1994.

[66] O. P. Popova, P. Jenniskens, and D. O. Glazachev, "Chelyabinsk meteoroid fragmentation," Dynamical Processes in Geospheres, vol. 5, pp. 59-78, 2014.

[67] D. D. Badyukov and A. E. Dudorov, "Fragments of the Chelyabinsk meteorite shower: distribution of masses and sizes and constraints on the mass of the largest fragment," Geochemistry International, vol. 51, no. 7, pp. 583-586, 2013.

[68] D. D. Badyukov, A. E. Dudorov, and S. A. Khaybrahmanov, "Mass distribution of fragments of the Chelyabinsk meteorite," Chelyabinsk Physical and Mathematical Journal, vol. 1, no. 330, pp. 40-46, 2014.

[69] M. Gritsevich, V. Vinnikov, T. Kohout et al., "A comprehensive study of distribution laws for the fragments of Košice meteorite," Meteoritics \& Planetary Science, vol. 49, no. 3, pp. 328-345, 2014.

[70] J. Moilanen, M. Gritsevich, and E. Lyytinen, "Determination of strewn fields for meteorite falls," Monthly Notices of the Royal Astronomical Society, vol. 503, no. 3, pp. 3337-3350, 2021.

[71] A. S. Betzler and E. P. Borges, "Mass distributions of meteorites," Monthly Notices of the Royal Astronomical Society, vol. 493, no. 3, pp. 4058-4064, 2020.

[72] V. A. Bronshten, Physics of Meteoric Phenomena, Springer, Dordrecht, Netherlands, 1983.

[73] S. Buhl and M. Baermann, The Bassikounou Meteorite Fall - Descriptive Catalog of the Recovered Masses https://www. meteorite-recon.com/wp-content/uploads/pdf/Buhl_ Baermann_Catalog, 2007.

[74] P. Jenniskens, M. H. Shaddad, D. Numan et al., "The impact and recovery of asteroid 2008 TC3," Nature, vol. 458, no. 7237, pp. 485-488, 2009.

[75] M. H. Shaddad, P. Jenniskens, D. Numan et al., "The recovery of asteroid 2008 TC3," Meteoritics \& Planetary Science, vol. 45, no. 10-11, pp. 1557-1589, 2010.

[76] J. Tóth, J. Svoreň, J. Borovička et al., "The Košice meteorite fall: recovery and strewn field," Meteoritics \& Planetary Science, vol. 50, no. 5, pp. 853-863, 2015.

[77] 2013 Database of the Chelyabinsk Meteorite Fragments of Chelyabinsk State University https://www.csu.ru/science/ chelyabinsk-meteor-study-center/database.aspx.

[78] The first regional https://www.1obl.ru/news/o-lyudyakh/fotovtorogzmeru-oskolka-meteorita-chelyabinsk/. 